\title{
Mitgestaltung in der kommunalen Sozialpolitik am Beispiel der Seniorenpolitik
}

Trotz gestiegener Wahlmöglichkeiten und Kundenorientierung nehmen Nutzer in der Sozialpolitik weiter auch eine Bürgerrolle ein. Die ehrenamtliche Mitgestaltung kommunaler Sozialpolitik ist ein Ausdruck davon. Aber welche Möglichkeiten haben bürgerschaftliche Interessenvertretungen, zwischen den Wahltagen ihre Stimme in kommunalen Entscheidungsprozessen zu erheben? Der Beitrag zeigt am Beispiel von Seniorenvertretungen, dass bürgerschaftliche Interessenvertretungen zwar wenig Vetopotenzial haben, aber kommunaler Sozialpolitik neue Impulse geben können. ${ }^{\circ}$

RALF OCH

\section{Einleitung}

Die wohlfahrtsstaatlichen Reformen der letzten Jahrzehnte führten zu einer stärkeren Ökonomisierung und Vermarktlichung sozialer Dienstleistungen (Evers/Heinze 2008; Le Grand 1991; Bode 2005) - Nutzer sozialer Dienstleistungen werden als Kunden adressiert (Eichler/PfauEffinger 2009; Vabø 2006). Macht die Steuerung über Marktmechanismen damit „Bürger“ und ihre Partizipation in politischen Entscheidungen überflüssig, da Entscheidungen nun zwischen verschiedenen Angeboten „mit den Füßen" und nicht mehr mit dem Stimmzettel oder dem Engagement in politischen Parteien und Gremien getroffen werden? Keineswegs, denn die Einführung von Choice (freie Wahl) hat die politischen Konflikte um Ressourcen und Ausgabenlevel öffentlicher Haushalte nicht beendet (Clarke 2006). Vielmehr nehmen Nutzer zwar eine Konsumentenrolle ein, wenn sie zwischen konkurrierenden Anbietern wählen (müssen), sie nehmen aber auch als Bürger auf das Angebot und seine Regulierung Einfluss (Ewert in diesem Heft; Clarke/Newman 2007). Es lässt sich also argumentieren, dass Sozialmärkte Bürger und Klienten nicht durch Konsumenten ablösen, sondern eine neue Aufgabenverteilung bzw. Gewichtung zwischen den Rollen stattgefunden hat (Clarke 2006). Während sich die anderen Beiträge dieses Schwerpunktheftes eher auf die Konsumentenrolle konzentrieren, wird im Folgenden der Schwerpunkt darauf gelegt, wie Nutzer in ihrer Bürgerrolle ihre Vorstellungen politisch artikulieren.

Zwei Möglichkeiten, als Bürger zu agieren, fallen sofort ins Auge: die Beteiligung an Wahlen und eher informelle und kurzzeitige öffentliche Meinungsbekundungen, wie sie in jüngster Zeit beispielsweise in den Auseinandersetzungen um Stuttgart 21, die Atompolitik oder die Occupy-Bewegung stark in den Fokus der Öffentlichkeit gerieten. Eine dritte Möglichkeit ist das längerfristige politische Engagement in Organisationen, etwa in politischen Parteien oder aber bürgerschaftlichen Interessenvertretungen.

In diesem Beitrag geht es um das politische Engagement von Seniorenvertretern in der kommunalen Sozialpolitik, insbesondere mit Blick auf kommunale Pflegepolitik. Senioren sind eine relativ heterogene Bevölkerungsgruppe, die eher selten im Fokus steht, aber derzeit schnell wächst. Den Älteren eine politische Stimme (Voice) zu geben, mag als eine zentrale Aufgabe einer alternden Gesellschaft erscheinen (Naegele 1999), da diese Gruppe als Wahl- wie auch als Kostenfaktor eine „zentrale Zielgruppe der Politik“ darstellt (Künemund 2001, S. 71) und über steigende „latente politische Macht“ (Schmidt 2009, S. 273) verfügt. Letztere wird

\footnotetext{
Ich danke ganz herzlich den Koordinatoren des Schwerpunktheftes und den Kollegen unseres Lehrstuhls, insbesondere Birgit Pfau-Effinger und Patricia Frericks, für ihre wertvollen Hinweise zur Überarbeitung des Beitrages.
} 
nicht nur über Wahlen („individual voice“), sondern auch über „collective voice“ (Haarmann et al. 2010, S. 217), d. h. Voice durch Interessenvertretungen, ausgeübt. Darunter fällt auch das Beispiel der hier analysierten Seniorenvertretungen. Sie sind parteipolitisch neutrale, konfessions- und verbandsungebundene Interessenvertretungen der (unscharf definierten) Lebenslage "Senioren“ gegenüber der Verwaltung, der Politik und allen weiteren relevanten Akteuren (Eifert 2006; Roth 1997). Im Idealfall wird durch sie also die Bürgerrolle der Nutzerinnen und Nutzer von sozialen Dienstleistungen realisiert.

Wenn man die Realisierung der Bürgerrolle untersuchen möchte, bieten sich Kommunen als Untersuchungsebene an, weil sich hier die Folgen gesamtgesellschaftlicher Trends zeigen und sich zugleich den Bürgern die Möglichkeiten bieten, „direkt in die politische Willensbildung und Entscheidungsfindung einzugreifen“ (Burchardt 2007, S. 8). Auch wenn kommunale Entscheidungen nicht das Gewicht landesoder gar bundespolitischer Beschlüsse haben, können sie in der Summe doch die lokalen Lebensverhältnisse erheblich beeinflussen. Gleichzeitig sind die Kommunen der Ort, an dem Nutzer als Bürger, Klienten und als Konsumenten auf staatliche Leistungen und deren Regelungen treffen, ob sie nun öffentlich bereitgestellt werden oder über den Markt.

Der Beitrag geht drei Fragen nach: Was sind die Spielräume für Seniorenvertretungen in kommunaler Sozialpolitik? In welchen Bereichen kommunaler Sozialpolitik üben Seniorenvertretungen Voice aus? Und schließlich, welche Zielsetzungen haben Seniorenvertretungen auf kommunale Sozialpolitik? Dabei wird auf das Konzept von Voice von Albert Hirschman (1970) zurückgegriffen.

Im Folgenden stelle ich zunächst die Anlage der zugrunde liegenden Studie vor (Abschnitt 2) und zeige im Anschluss daran, wie das Voice-Konzept in politischen Kontexten wirkt (3). Der vierte Abschnitt umreißt die Voice-Spielräume, die kommunale Seniorenvertretungen haben. In den beiden folgenden Abschnitten zeige ich, in welchen sozialpolitischen Bereichen (5) und mit welchen Zielsetzungen (6) die Seniorenvertreter ihre Voice-Spielräume nutzen. Zuletzt fasse ich die Ergebnisse mit dem Ziel zusammen, allgemeinere Erkenntnisse über das Potenzial dieser Form einer ausgefüllten Bürgerrolle der Nutzerinnen und Nutzer zu gewinnen (7).

\section{Untersuchungsanlage}

Die drei Forschungsfragen werden auf Grundlage von explorativ vergleichenden Fallstudien in vier mittelgroßen Städten zwischen 75.000 und 100.000 Einwohnern beantwortet. Die Fallstudien entstanden im Rahmen des von der Deutschen Forschungsgemeinschaft (DFG) geförderten Projekts „Die lokale Restrukturierung der Altenpflege - kulturelle Grundlagen, Akteure und Handlungsbedingungen“. Je zwei Städte liegen in West- bzw. Ostdeutschland; das Aus- wahlkriterium war, möglichst ähnliche Städte zu finden (similar cases design), was hinsichtlich Größe, Alterungsdruck und kommunaler Haushaltsgröße weitestgehend gelang. W 1 liegt in Hessen, W 2 in Niedersachsen, O 1 in Thüringen und $\mathrm{O} 2$ in Sachsen-Anhalt. ${ }^{{ }^{3}}$

Insgesamt wurden 38 Interviews mit Experten der lokalen Politik und Zivilgesellschaft durchgeführt und nach den Maßgaben der qualitativen Inhaltsanalyse von Mayring (2008, 2000) ausgewertet. Darunter waren alle Sozialdezernenten, ${ }^{\circledR}$ alle Vorsitzenden der Sozialausschüsse, die für die lokale Pflegepolitik verantwortlichen Verwaltungsvertreter in den Städten sowie weitere politisch relevante lokale Akteure wie Anbieter oder Seniorenvertreter. Darüber hinaus wurden die für die Seniorenvertreter maßgeblichen institutionellen Regelungen sowie die Protokolle der Sozialausschüsse von 1995- 2007 inhaltsanalytisch ausgewertet, um die Entwicklung seniorenrelevanter Themen und die Partizipation der Seniorenvertreter in diesen Policy-Debatten nachvollziehen zu können.

\section{Voice in politischen Kontexten}

Ursprünglich hatte Hirschman (1970) sein Konzept von Exit, Voice und Loyality entwickelt, um zu zeigen, wie unzufriedene Kunden mit den Anbietern von Gütern und Leistungen umgehen können. Dafür unterscheidet er die zwei grundlegenden Mechanismen Exit und Voice. Exit (Abwanderung) meint schlicht, dass sich unzufriedene Kunden einen neuen Anbieter suchen, der eine äquivalente Leistung offeriert - der klassische Marktmechanismus. Wenn aber ein äquivalenter Anbieter nicht verfügbar ist oder ein Wechsel (höhere) Kosten verursacht, kann Voice ${ }^{\ominus}$ - das Kundtun der Unzufriedenheit - eine sinnvolle Alternative sein, um den Anbieter zu veranlassen, sein Produkt zu verbessern. Voice ist damit ein eminent politischer Mechanismus

(2) Das Projekt wurde zwischen März 2006 und Juni 2011 unter der Leitung von Birgit Pfau-Effinger und unter Mitarbeit von Melanie Eichler und dem Autor an der Universität Hamburg durchgeführt. Ziel des Projektes war ein Vergleich lokaler Pflegepolitiken zwischen ost- und westdeutschen Städten, der hier keine große Rolle spielt (Pfau-Effinger et al. 2012).

(3) Eine der Fallstädte sagte ihreTeilnahme an der Studie nur unter der Bedingung zu, dass sie nicht namentlich genannt wird, was selbstverständlich respektiert und analog auch für die anderen Fallstädte eingehalten wird.

(4) Außer in W 2. Der zum Erhebungszeitraum zuständige Sozialdezernent war nicht für ein Interview zu gewinnen und verwies uns an seinen zuständigen Verwaltungsmitarbeiter.

(5) In der deutschen Fassung von 1974 wurde der Begriff m. E. etwas eng als Widerspruch übersetzt. 
(ebd., S. 15). Die Wahrscheinlichkeit, mit der Voice gegenüber Exit auftritt, hängt vom Grad der Loyalität zu dem Anbieter ab, die Hirschman als Funktion der Exitoptionen bzw. -kosten modelliert. Die Loyalität hängt also von dem Kalkül ab, welchen Gewinn ein Anbieterwechsel bringen würde. Ohne Exitoptionen, also die Möglichkeit den Anbieter zu wechseln, gibt es aber auch keine Voice-Möglichkeiten, da letztlich eine Unmutsäußerung nur dann gehört wird, wenn glaubhaft eine Abwanderung angedroht werden kann.

In politischen Kontexten ist der Grad der so verstandenen Loyalität naturgemäß hoch, da das Verlassen eines politischen Gemeinwesens in aller Regel erhebliche Kosten mit sich bringt und darüber hinaus den Wirkungen politischen Handelns oft dennoch nicht entgangen werden kann (ebd.). Voice in politischen Kontexten ist kostspielig, im Erfolg unsicher und erfordert oft ein langfristiges Engagement (Crouch 1995, S. 67). Ob sich Nutzer nun passiv verhalten und die negativen Effekte von Politiken akzeptieren oder aber zu aktiven Bürger werden und Voice ausüben (Rokkan 1974, S. 49), hänge daher wesentlich davon ab, wie sie den "trade-off“ zwischen den Nachteilen der bestehenden oder künftigen Politiken und den Erfolgsaussichten für deren Veränderung einschätzen (Hirschman 1970, S. 77).

\section{Voice-Spielräume für kommunale Seniorenvertretungen}

Welche Spielräume hat kommunale Sozialpolitik im föderalen Rahmen der Bundesrepublik und welche Stellung können Nutzer - hier in Form von Seniorenvertretungen - in der Gestaltung kommunaler Sozialpolitik einnehmen? Die großen Linien der Sozialpolitik werden in der Bundesrepublik zwar von Bund und Ländern vorgegeben. Kommunale Sozialpolitik hat aber im Rahmen der vom Grundgesetz garantierten kommunalen Selbstverwaltung einen eigenen Stellenwert und ist eine „wesentliche Angelegenheit der örtlichen Gemeinschaft [und] ein eigenständiges kommunales Politikfeld“ (Backhaus-Maul 1994, S. 529).

Dabei wird zwischen zwei zentralen Aufgabenbereichen unterschieden (Backhaus-Maul 1998; Dahme/Wohlfahrt 2011a): Pflichtaufgaben, die aus den rechtlichen Vorgaben und/oder Aufgabenübertragungen des Bundes bzw. der Länder entstehen, und die aus dem Selbstverwaltungsrecht erwachsenden freiwilligen Aufgaben. Mit Blick auf die Pflichtaufgaben ist kommunale Sozialpolitik vor allem Implementationspolitik mit mehr oder minder großen Spielräumen (Dahme/Wohlfahrt 2011b, S. 395). Hinsichtlich der freiwilligen Aufgaben können die Kommunen „sozialpolitisch scheinbar unbegrenzt gestaltend aktiv werden" (Roth 1999, S. 3), was praktisch allerdings durch die verfügbaren Ressourcen erheblich eingeschränkt wird (Dahme/Wohlfahrt 2011a).
Backhaus-Maul (1998) sieht die Gestaltungsspielräume der Kommunen in der Sozialpolitik in der Festlegung von Menge und Qualitätsstandards der sozialen Leistungen, der Gestaltung der Trägerlandschaft und dem Angebot an sozialen Diensten. Im Anschluss an Pabst (2002) lassen sich die verschiedenen Aufgaben kommunaler Sozialpolitik in fünf Gruppen von Maßnahmen unterteilen: Planungsmaßnahmen, Infrastrukturmaßnahmen, Maßnahmen zur Erhaltung/Verbesserung der Qualität sozialer Dienstleistungen, Preisgestaltung sozialer Dienstleistungen, Beratungs- und Informationsmaßnahmen.

Wie in Bund und Ländern wird Sozialpolitik in den Kommunen in drei Arenen gestaltet: in der Öffentlichkeit, dem Stadtparlament und der Verwaltung (Pitschas 2000, S. 130). In politischen Entscheidungsprozessen auf der kommunalen Ebene sind vor allem das Stadtparlament und die Verwaltung zentral, während öffentliche Diskurse meist von geringerer Bedeutung sind (Backhaus-Maul 1998, S. 699; Knemeyer 1995).

In diesen Arenen agieren drei größere Gruppen von Akteuren: die Vertreter der lokalen (Partei-)Politik, die Verwaltungen und die sehr heterogene Gruppe zivilgesellschaftlicher Akteure. Die Politik hat durch ihr Budgetrecht eine Vetoposition, während der professionellen Verwaltung ein Wissensvorsprung gegenüber den meist ehrenamtlichen Politikern zugesprochen wird.

Zur Gruppe der zivilgesellschaftlichen Akteure gehören auch Seniorenvertretungen. ${ }^{\circledR}$ Sie wurden in den letzten Jahrzehnten in allen Teilen des Landes und auf allen politischen Ebenen gegründet (Mayer 2000). In ihrem Artikel zu Seniorenvertretungen in Nordrhein-Westfalen beschreibt Barbara Eifert (2006, S. 268ff.) vier zentrale Aufgaben, die für alle kommunalen Seniorenvertretungen gelten können: Mitwirkung bei kommunalen Planungen, Beratung von Politik, Verwaltung sowie altenpolitischen Akteuren, Vermittlung und Beratung älterer Menschen, Öffentlichkeitsarbeit.

Auf der kommunalen Ebene sind Seniorenvertretungen zumeist freiwillige Einrichtungen, d. h. die Kommune kann, muss sie aber nicht einrichten. ${ }^{\circledR}$ Als institutionalisierte Interessenvertretungen haben sie Voice-Möglichkeiten, die ihnen durch die politischen Akteure zugestanden werden, weil sie die Legitimität der Interessenvertretung anerkennen (müssen) oder aber ein Eigeninteresse daran haben. Sie können formell oder informell sein; inwieweit die formalisierten Voice-Rechte über die eines normalen Bürgers hinausgehen, bleibt eine empirische Frage.

6 Dazu zählen beispielsweise Seniorenbeiräte, Seniorenräte, Seniorenbeauftragte, Seniorenausschüsse, Altenrat, Altenbeirat, Stadtaltenring (Mayer 2000).

(7) Die hier untersuchten Seniorenvertretungen haben ihre Voice-Rechte durch die jeweiligen Stadtparlamente erhalten, da es keine bundesweit und außer in Schleswig-Holstein (Vanselow 2000, S. 123) auch keine landesweit geregelten Beteiligungsrechte gibt. 
Dadurch gibt es aber auch eine große Bandbreite von formalisierten Voice-Rechten (vgl. für Nordrhein-Westfalen Eifert 2006). Auch zwischen den hier untersuchten Fällen sind die Unterschiede erheblich. Zwar sind alle untersuchten Seniorenvertretungen zumindest im Sozialausschuss ohne Stimmrecht vertreten, aber über diesen kleinsten gemeinsamen Nenner hinaus haben die Seniorenvertreter in Kommune O 1 die stärksten Voice-Rechte. Sie verfügen in den politischen Entscheidungsprozessen über umfassende Antrags- und Informationsrechte und ihre Einlassungen verpflichten Stadtrat und Verwaltung zu einer zeitlich festgelegten Reaktion. Etwas schwächer sind die Voice-Rechte des Seniorenbeirats in Kommune W 1 zu sehen, der zwar in allen Seniorenfragen zu beteiligen ist, aber im Stadtrat nur Anhörungs- und keine Antragsrechte hat, die Stadtrat oder Verwaltung zur Reaktion verpflichten. Ähnlich hoch sind auch die Voice-Rechte in Kommune O 2 ausgestaltet, die sich im Unterschied zu Kommune O 1 aber auf den Sozial-, Kultur- und Bildungsausschuss beschränken. Die Beteiligung der Seniorenbeauftragten in Kommune $\mathrm{O} 2$ an Verwaltungsvorgängen erscheint aufgrund einer organisatorischen Anbindung der Seniorenbeauftragten an das Sozialdezernat höher als in Kommune W 1. Die geringsten Voice-Rechte hat die Seniorenvertretung in Kommune W 2, die nur einen Vertreter in den Sozialausschuss entsenden kann, der dort aber nur Rederecht und kein Antragsrecht und nur einen sehr vage formulierten Zugang zu den Verwaltungsprozessen hat.

\section{Sozialpolitische Bereiche der Voice-Ausübung}

Die formalisierten Voice-Rechte sind nur eine Seite der Medaille, ihre Ausübung die andere. Die im Folgenden zu klärende Frage ist daher, in welchen Bereichen die Seniorenvertretungen ihre Voice-Möglichkeiten nutzen, um die kommunale Sozialpolitik ${ }^{\circledR}$ in den untersuchten Städten zu beeinflussen.

\subsection{Voice im Bereich kommunaler Planung}

Der Fokus kommunaler Planung hat sich mit der Einführung der Pflegeversicherung 1995/96 verschoben. Während vor 1995 das Ziel die Informationsgewinnung für die Steuerung der Bedarfsdeckung und der Preise war - die klassische Altenhilfeplanung (siehe Klie/Pfundstein 2008), so geht es heute um die Moderation von Planungsprozessen zur Innovationsförderung und Vernetzung sowie um Marktbeobachtung, beispielsweise mittels Förderung der Infrastruktur und Pflegekonferenzen (ebd., S.7). Allerdings führte eine der untersuchten Kommunen (O 1) im Beobachtungszeitraum keine Planungsmaßnahmen durch.
In der Kommune W 1 war die Erstellung einer neuen Altenhilfeplanung eine der zentralen Maßnahmen in der Seniorenpolitik, der Seniorenbeirat war hier wenig beteiligt. Kommune W 2 führt jährlich zwei bis drei Pflegekonferenzen durch, die der Marktbeobachtung und der Besetzung vor allem qualitätsrelevanter Themen im Bereich der Pflege (wie Demenzbetreuung oder Wohnkonzepte für Senioren) dienen. An ihnen nahmen die Seniorenvertreter teil - wenn auch passiv - (Sozialamt W 2 Abteilungsleiter Altenhilfe). Die Kommune O 2 hat seit der Einführung der Pflegeversicherung zwei kommunale Altenhilfeplanungen durchgeführt, in die Seniorenvertreter einbezogen waren.

\subsection{Voice im Bereich kommunaler Infrastrukturmaßnahmen}

Hinsichtlich des finanziellen Umfanges gehören Infrastrukturmaßnahmen in der Regel zu den bedeutenderen Maßnahmen in der kommunalen Sozialpolitik. Als Infrastruktur wird hier die bauliche Substanz der Einrichtungen sowie die inhaltliche Ausrichtung von Organisationen verstanden (stationär/teilstationär/ambulant, Spezialisierungen wie Demenzbetreuung u. ä.).

Alle vier untersuchten Städte unterhalten als freiwillige Aufgabe jeweils sechs bis zehn Seniorenfreizeitstätten, in denen Beratungen zu verschiedenen seniorenbezogenen Themen wie Pflege, Wohnen oder Sozialhilfe von Vereinen oder den Seniorenbeiräten selbst oder kulturelle Veranstaltungen angeboten werden. In den Fällen, in denen sie von den lokalen Wohlfahrtsverbänden betrieben werden, erhalten sie einen Zuschuss zu den Unterhaltungs- und Personalkosten. Die Seniorenfreizeitstätten waren in den beiden westdeutschen Städten offenbar nie umstritten. In der Kommune O 1 gelang es, die sieben Seniorenfreizeitstätten, die über einen längeren Zeitraum hälftig von der Stadt und vom Land finanziert wurden, ohne Kürzungen in den Stadthaushalt zu überführen. Darüber hinaus unterstützt die Kommune die Ausbildung von Seniorenbegleitern. In der Kommune O 2 war im Zusammenhang mit der Diskussion um Kürzung des Sozialhaushaltes auch die Gesamtzahl der acht Seniorenfreizeitstätten infrage gestellt worden. Der Verwaltung gelang es aber zusammen mit der Seniorenvertretung und Teilen der Politik, diese zu erhalten (Protokolle Sozialausschuss O 2 2002). Die Seniorenvertretung hat hier die Ausbildung von Seniorenbegleitern angestoßen und koordiniert auch deren Einsatz.

Die Sicherstellung der pflegerischen Infrastruktur ist eine Pflichtaufgabe, die das Pflegeversicherungsgesetz (SGB XI) in $\S 8$ den Ländern und Kommunen zuweist. Dazu können sie den Anbietern die Kosten des Auf- oder

8 Die Beschränkung auf das Feld der Sozialpolitik, insbesondere Pflegepolitik, klammert allerdings weitere wesentliche Interessenfelder der Seniorenpolitik, wie beispielsweise in den Bereichen Verkehrs- oder Kulturpolitik, aus. 
Umbaus von Pflegeheimen teilfinanzieren, die dafür eine gewisse Zeit lang diesen Investitionskostenanteil nicht auf die Pflegebedürftigen umlegen dürfen. Nur in der Kommune $\mathrm{O} 2$ hatte die Stadt die Möglichkeit, die dafür vorgesehenen Landesmittel selbst zu vergeben. Die Mittel wurden förmlich ausgeschrieben und im „richtig demokratischen Weg“" (Leitung Sozialamt O 2) durch die Gremien gebracht. Die Kommune erreichte das von den Seniorenvertretern unterstützte Ziel, fast $45 \%$ der damals vorhandenen Betten für zehn Jahre auf einen niedrigeren Preis zu binden (in der Pflegestufe I durchschnittlich 40 \%) (Leitung Sozialamt O 2).

Bis auf W 1 unterhalten bzw. unterhielten alle Kommunen eigene stationäre Pflegeeinrichtungen. Das kleinste der drei stationären Heime in der Kommune O 2 wurde 1998 aufgrund zu hoher Sanierungskosten geschlossen. Die zentralen Verhandlungspartner waren hier der Stadtrat, die zuständigen Ämter und die Personalvertretung. Der Seniorenbeirat drang aber erfolgreich darauf, die Bewohner nach ihren bestehenden Wohngruppen auf die beiden anderen kommunalen Heime zu verteilen. Diese beiden größeren Heime wurden mit Bundes- und Landesmitteln saniert und 2000 in einem öffentlichen Ausschreibungsverfahren an lokale Wohlfahrtsverbände verkauft. Der Seniorenbeirat plädierte erfolglos für einen Anbieter mit demenzfreundlichem Wohnkonzept (O 1 Altenhilfeplanerin, Protokolle Sozialausschuss). Die Seniorenbeiräte in den Kommunen $\mathrm{O} 1$ und $\mathrm{O} 2$ engagierten sich darüber hinaus für den Ausbau betreuten Wohnens, was in beiden ostdeutschen Städten ein weitaus größeres Thema war als in den Westdeutschen. Die Kommune O 2 besaß im Untersuchungszeitraum ein Pflegeheim, das zunächst auch verkauft werden sollte, aber nach einer längeren Diskussion bei der Stadt verblieb. Es wurde aufwendig saniert und auf Demenzbetreuung spezialisiert. Auch hier waren die entscheidenden Akteure der Stadtrat, die zuständigen Ämter und der Personalrat. Die Seniorenvertreter blieben eher marginal, unterstützten aber die Demenzausrichtung. Sie engagierten sich auch für die Einrichtung einer Tagesbetreuung für Demente und erreichten die Aufnahme dieses Zieles in die Fachplanung. Ein weiterer, zum Untersuchungszeitpunkt noch nicht beendeter Prozess war die Einrichtung eines stationären Hospiz in der Stadt, das weitgehend auf eine Initiative zivilgesellschaftlicher Kräfte (Wohlfahrtsverbände, Vereine) inklusive der Seniorenvertreter zurückging und dem die Stadt sehr wohlwollend gegenüberstand.

\subsection{Voice in der Gestaltung der Preise sozialer Dienstleistungen}

Die Beeinflussung von Preisen für die Inanspruchnahme sozialer Dienstleistungen ist für die Kommunen am einfachsten steuerbar, wenn sie diese als freiwillige Leistungen selbst erbringen: Der Besuch der oben beschriebenen Seniorenbegegnungsstätten war in allen unseren Städten für die Bürger kostenfrei. Die Preise für Pflichtaufgaben können die Kommunen meist kaum beeinflussen, wie das Beispiel der Pflege verdeutlicht: Die Pflegeversicherung als „Teilkaskoversicherung "kommt nur für einen Teil der Kosten auf, den Rest müssen die Pflegebedürftigen aufbringen. Die Kommunen haben zwar ein genuines Interesse an niedrigen Preisen, da sie für die Sozialhilfeempfänger aufkommen müssen. Gleichzeitig haben sie aber nur eine begrenzte Verhandlungsmacht, da in allen beobachteten Fällen die Pflegeentgelte in landesweiten Verhandlungskommissionen ausgehandelt werden. Eine Ausnahme bilden die oben schon erwähnten Investitionskosten, sofern sie auf kommunaler Ebene verhandelt werden können.

In der Kommune W 2 haben die öffentlichen Akteure die Regeln für die Investitionskostenförderung so angepasst, dass ältere Einrichtungen etwas günstiger wurden. Darüber hinaus hat die Kommune mit den Pflegekassen für zwei Einrichtungen mit spezieller Demenzbetreuung höhere Pflegesätze ausgehandelt, sodass sie für Pflegebedürftige im Vergleich zu anderen Einrichtungen nicht teurer wurden. Der Seniorenbeirat war zu keinem Zeitpunkt involviert. In der Kommune O 1 hat der Seniorenbeirat längere Zeit vergeblich darauf hingearbeitet, für den öffentlichen Nahverkehr nicht nur ein Sozial-, sondern auch ein vergünstigtes Seniorenticket einzuführen. In O 2 konnte die Kommune durch die Vergabe von Investitionskostenförderungen - wie oben gesagt - die Kosten in der stationären Pflege eine Zeit lang begrenzen.

\subsection{Voice im Bereich der Qualität sozialer Dienstleistungen}

Von zentraler Bedeutung ist die Qualität in der Pflege und im betreuten Wohnen für Senioren mit Betreuungs- und/ oder Pflegebedarf. Allerdings haben die einschlägigen rechtlichen Regelungen (vor allem Sozialgesetzbuch (SGB) XI und Heimgesetz) den Kommunen wenig Kompetenzen an die Hand gegeben, steuernd in die Qualität der Leistungserbringung in der Pflege einzugreifen, da hierfür die Heimaufsicht und der Medizinische Dienst der Krankenkassen (MDK) zuständig sind. Die Kommunen können aber zusätzliche Mittel für die Verbesserung der Qualität bereitstellen bzw. entsprechende Maßnahmen anbieten.

Die Kommune W 2 hat seit den 1980er Jahren einen institutionalisierten Diskurs über Pflegequalität, darüber hinaus ist W 2 die einzige Stadt in unserem Sample, in der die Heimaufsicht bei der Stadt angesiedelt ist und die so zumindest in der stationären Pflege selbst steuernd eingreifen kann. Der Seniorenbeirat bezog aber im Untersuchungszeitraum, nicht nur in der Diskussion um Pflegequalität, kaum Stellung. In der Kommune O 1 ist der Seniorenbeirat sehr aktiv und versucht bspw. mit den stationären Einrichtungen Partnerschaften zu schließen, um den Bewohnern ein über den Heimbeirat hinausreichendes Sprachrohr zu sein. Darüber hinaus hat er den einzigen städtischen Pflegeskandal publik gemacht. Die Seniorenvertretung in der Kommune O 2 unterstützte, wie oben gesagt, die Demenzausrichtung des kommunalen Heimes, nicht zuletzt, weil 
Zielsetzungen von Voice kommunaler Seniorenvertretungen in der Sozialpolitik

\begin{tabular}{|c|c|c|c|}
\hline & $\begin{array}{l}\text { Entscheidungen verhindert/ } \\
\text { Entscheidungsrichtung verändert }\end{array}$ & Neue Impulse gegeben & Bestehende Impulse verstärkt \\
\hline Planung & & & O 2, W 1 Seniorenhilfeplanung \\
\hline $\begin{array}{l}\text { Infrastruktur- } \\
\text { maßnahmen }\end{array}$ & $\begin{array}{l}\text { O } 2 \text { Anzahl Seniorenbegegnungsstätten } \\
\text { O } 1 \text { Kosten Seniorenbegegnungsstätten }\end{array}$ & $\begin{array}{l}\text { O } 2 \text { stationäres Hospiz } \\
\text { O } 2 \text { Ausbildung/Einsatz Seniorenbegleiter } \\
\text { O } 2 \text { Tagesbetreuung Demenz }\end{array}$ & $\begin{array}{l}\text { O } 2 \text { Demenzorientierung kommunales Heim } \\
\text { O } 2 \text { Investitionskosten } \\
\text { O } 1, \text { O } 2 \text { betreutes Wohnen } \\
\text { O } 1 \text { Ausbildung Seniorenbegleiter }\end{array}$ \\
\hline Qualität & & $\begin{array}{l}\text { O } 1 \text { Verbleib Pflegebedürftiger bei } \\
\text { Heimschließßung } \\
\text { O } 1 \text { Qualitätsskandal }\end{array}$ & \\
\hline Preise & & O 1 Seniorenticket & O 2 Investitionskostenzuschüsse \\
\hline Seniorenberatung & & O 1, O 2 Seniorentage & W 1, O 1 Seniorenratgeber \\
\hline Politikberatung & & O 2 Petitionen an Landtag und Bundestag & \\
\hline
\end{tabular}

Quelle: Darstellung des Autors.

sie die Qualität der Ausbildung insbesondere in der Demenzbetreuung als unzureichend ansah.

\subsection{Voice im Bereich kommunaler Beratungsleistungen}

Beratungsleistungen sind eine Kernaufgabe der Seniorenvertretungen (Eifert 2006, S.267), aber auch der Kommunen. Beide weisen, wie die nachfolgende Darstellung zeigt, eine sehr große Bandbreite in Angebot und Umsetzung auf.

Eine zentrale Beratungsstelle, bei der die Bürger alle Informationen rund um Pflege aus einer Hand erhalten, hat die Kommune in W 1 in Zusammenarbeit mit den Wohlfahrtsverbänden aufgebaut. An ihrer Steuerung ist die Seniorenvertretung nur marginal beteiligt. Die übrigen drei Städte haben ihre Seniorenberatungen thematisch und/oder räumlich stärker dezentralisiert. In der Kommune W 2 ergänzt der Seniorenbeirat das über die Stadt verstreute Angebot an Beratungsstellen der Stadt in Abstimmung mit der Verwaltung durch eine telefonische Seniorenberatung. In O 1 organisiert der Seniorenbeirat in Zusammenarbeit mit der Stadt die Seniorentage, eine jährlich stattfindende Veranstaltungsreihe, bei der sich praktisch alle mit Senioren befassten Akteure in der Stadt treffen und ihre Aktivitäten vorstellen. Dazu kommt ein „Mitmachtag“, der Seniorenwegweiser, die Seniorenzeitschrift und der „Wegweiser Demenz", die alle in Zusammenarbeit mit der Stadt organisiert werden. Ähnlich ist die Situation in der Kommune O 2, wo die Seniorenvertreterin zusammen mit der Stadt und dem Seniorenbeirat die jährliche Seniorenwoche organisiert und den Seniorenratgeber produziert. Darüber hinaus haben die Seniorenvertreter im Beobachtungszeitraum eine Stellungnahme und eine Petition an die Landesregierung von Sachsen-Anhalt bzw. das Bundesministerium für Gesundheit eingereicht.

\section{Zielsetzungen der Voice-Ausübung}

Seniorenvertretungen üben Voice in praktisch allen Bereichen kommunaler Sozialpolitik aus. Die Schwerpunkte in den beobachteten Fällen liegen, wie die Übersicht 1 zeigt, im Bereich von Infrastrukturmaßnahmen und Qualitätssicherung, was aber auch von den sozialpolitischen Schwerpunktsetzungen der jeweiligen Kommunen abhängig ist.

Im Folgenden frage ich nun nach den Zielsetzungen der Voice-Ausübung kommunaler Seniorenvertretungen. Dabei geht es weniger um die tatsächlich erreichten Wirkungen, denn um diese zu analysieren, müssten die Einflüsse anderer Akteure sehr viel stärker einbezogen werden, als dies im Rahmen dieses Beitrags möglich ist. Vielmehr soll es vor allem um die intendierten Wirkungen gehen, die über drei Indikatoren gemessen werden:

(1) Kommunale Entscheidungen verhindern oder ihre Richtung verändern. Dabei versucht die Seniorenvertretung, die Weiterführung einer bestehenden Maßnahme oder eine geplante Maßnahme zu verhindern, bzw. eine bestehende oder geplante Maßnahme in ihrem Charakter oder ihrem Umfang zu verändern.

(2) Neue Impulse geben. Hier versucht die Seniorenvertretung neue Ideen oder Maßnahmen anzuregen, unabhängig vom Erfolg des Impulses.

(3) Impulse verstärken. Die Seniorenvertretung versucht, bestehende oder geplante Maßnahmen zu unterstützten.

Übersicht 1 fasst die Bereiche, in denen Seniorenvertretungen Voice ausüben, und die Zielsetzungen zusammen. 
Aus Platzgründen können im Folgenden nicht alle in der Übersicht aufgeführten Voice-Aktivitäten diskutiert werden. Ich werde mich auf ausgewählte Fälle beschränken, die das Spektrum der Aktivitäten widerspiegeln und darüber hinaus eine vorsichtige Interpretation der Gründe des Erfolgs oder Misserfolgs dieser Voice-Ausübungen geben.

\subsection{Entscheidungen verhindert/Richtung der Entscheidung verändert}

Ein Beispiel für die Verhinderung oder Richtungsveränderung kommunaler Entscheidungen ist der oben schon angeführte Fall der Finanzierung der Seniorenfreizeitstätten in der Kommune O 1. Noch vor Auslaufen der Landesförderung über $50 \%$ der Kosten im Jahr 2004 initiierte die Altenhilfeplanerin der Stadt zusammen mit dem Sozialdezernenten und dem Seniorenbeirat die vollständige Kostenübernahme durch die Kommune.

„Das war auch ein Punkt in meiner Amtszeit als Sozialdezernent, wo wir die Summe mal verdoppelt haben, um die es da ging, in einem unbeobachteten Moment, wo der Stadtrat gar nicht gemerkt hat, dass wir mehr Geld eingestellt haben“" (ehemaliger Sozialdezernent O 1).

Die entscheidende Rolle spielte aber nicht der Seniorenbeirat, sondern die Verwaltung sowie der Sozialdezernent. Da die Seniorenvertreter kein Stimm- oder gar Vetorecht im Stadtrat haben, benötigen sie stets die Unterstützung durch Akteure in der Politik und meist auch der Verwaltung. Auch im zweiten oben genannten Fall, der Diskussion um die Verringerung der Anzahl der Seniorenfreizeitstätten in der Kommune O 2, waren die Seniorenvertreter auf die Unterstützung des Sozialausschusses angewiesen (Protokolle Sozialausschuss Dezember 2002).

\subsection{Neue Impulse}

$2005 \mathrm{kam}$ es in einem der privatwirtschaftlichen Heime in der Stadt O 1 zu Verbrühungen von zwei Bewohnern durch falsch eingestellte Duscharmaturen. Der Seniorenbeirat erfuhr davon und informierte das Sozialamt und die lokale Presse. Darüber hinaus beantragte er die Behandlung der Vorfälle im Sozialausschuss (Protokoll Sozialausschuss, Dezember 2005).

„Es ist angezeigt worden vom Seniorenbeirat. Es gibt ja in O 1 ein vernünftiges Netzwerk. Sobald Fehlentwicklungen auftreten, werden die dann auch öffentlich, nicht nur über die Presse, sondern auch über Beiräte z. B. Seniorenbeirat, der ja sehr aktiv ist und sehr gut funktioniert in $\mathrm{O} 1$. Und dann sind die Wege kurz und es kommt dann sofort in den Sozialausschuss und auf die Tagesordnung, die dann auch besprochen wird. Wir haben dann die Betroffenen eingeladen, eine kleine Anhörung dann durchgeführt. Haben wir zumindest gemacht mit den Vertretern des Heimes, der Pflegeeinrichtung, mit dem, jetzt fällt mir gerade die Abkürzung nicht ein, die Kontrollbehörde heißt MDK“ (Vorsitzender Sozialausschuss).
Im Ergebnis wurden mit dem Betreiber des Heimes Qualitätssicherungsmaßnahmen und ein Bericht des Betreibers darüber vereinbart. Der Fall ist insofern sehr bemerkenswert, als es dem Seniorenbeirat gelungen ist, die Kommune in der Sicherung der Pflegequalität zu aktivieren, wofür die Kommunen i.d.R. keine Kompetenzen haben. In den anderen untersuchten Städten ohne kommunale Heimaufsicht (W 1; O 2) verwiesen die kommunalen Akteure auch auf ihre eigenen geringen Handlungsmöglichkeiten in solchen Fällen.

Ein Beispiel für die Beeinflussung von Preisen sozialer Dienstleistungen ist der Versuch des Seniorenbeirats der Kommune O 1, vergünstigte Seniorentickets in der Stadt einzuführen. Es gelang ihm zunächst, den Sozialausschuss dafür zu gewinnen. Das Anliegen scheiterte letztlich aber am lokalen Verkehrsverbund, der darauf hinwies, dass es im Widerspruch zum Gleichbehandlungsgrundsatz des Personenbeförderungsgesetzes stünde und dass es bereits ein Sozialticket gebe, das ca. 300 Rentner der Stadt nutzten (Protokolle Sozialausschuss Mai-August 1996).

Die Seniorenvertretung der Kommune O 2 reichte im Untersuchungszeitraum mehrere Stellungnahmen oder Petitionen zu aktuellen seniorenpolitischen Fragen beim Landtag in Sachsen-Anhalt oder dem Bundestag ein und ging so über die kommunalpolitische Ebene hinaus. Besonders interessant ist die 2007 verabschiedete Petition an den Bundestag zur Reform der Pflegeversicherung, weil sie dafür die Unterstützung des Sozialausschusses erreichte. Die Seniorenvertretung plädierte darin für eine stärkere Förderung von Prävention, eine Stärkung der Kompetenzen in der Familienpflege sowie eine Verbesserung der Berufs- und Weiterbildung. Demenzerkrankungen sollten in die Pflegeversicherung einbezogen werden und die Qualitätsnachweispflicht auch auf Angebote betreuten Wohnens ausgeweitet werden (Protokolle Sozialausschuss April 2007).

\subsection{Bestehende Impulse verstärken}

Altenhilfeplanungen sind Aufgaben der Kommune und ein sehr geeigneter Fall für Voice-Ausübung von Seniorenvertretungen. Allerdings kann Voice, wie die beiden nachfolgenden Beispiele zeigen, von den Seniorenvertretungen sehr unterschiedlich ausgeübt werden.

Die Erneuerung der Altenhilfeplanung war eine der größten Maßnahmen, die die Kommune W 1 in unserem Untersuchungszeitraum durchführte. Maßgeblich beteiligt daran waren die in der Steuerungsgruppe Altenhilfeplanung zusammenarbeitenden Vertreter kommunaler Politik und Verwaltung und die lokalen Wohlfahrtsverbände. Der Seniorenbeirat war nicht Mitglied der Steuerungsgruppe. Er wurde ab und an über den Stand der Planungen informiert, ohne aktiv an der Planung teilzuhaben.

„[...] die Altenhilfeplanung wird sehr von der Politik gesteuert, [...] da steckt auch immer das Geld mit dahinter. Heute [...] wird nicht mehr, sag ich jetzt einfach mal, nach Bedarf und Nachfrage entschieden, sondern nur nach Haushaltslage" (Leiterin Beratungs- und Koordinationsstelle). 
Demgegenüber waren die Seniorenvertreter der Kommune O 2 von Anfang an aktiv in die Gestaltung der Altenhilfeplanung der Stadt einbezogen, an der darüber hinaus eine ganze Reihe weiterer Akteure wie die Behindertenbeauftragte, das Kuratorium Deutsche Altenhilfe, das Sozialamt, die Stadtentwicklung und andere mitwirkten (Protokolle Sozialausschuss Mai 1997; Pflegestrukturplan 1999, 2001).

Die oben bereits ausführlich beschriebenen kommunalen Beratungsleistungen zeigen ebenfalls, dass Voice in der Verstärkung oder Unterstützung einer kommunalen Maßnahme durch die Seniorenvertretungen sehr unterschiedliche Grade annehmen kann.

\section{Fazit}

Der vorliegende Beitrag fragt nach drei Aspekten der Nutzerbeteiligung durch Seniorenvertretungen: Spielräume für Seniorenvertretungen in kommunaler Sozialpolitik, den Bereichen kommunaler Sozialpolitik, in denen Seniorenvertreter Voice ausüben, und den Zielsetzungen ihrer Voice-Ausübung.

Aufgrund ihrer Stellung als ,vorparlamentarische Interessenvertretungen" (von Alemann 2000) sind Seniorenvertretungen darauf angewiesen, dass ihnen von der kommunalen Politik Voice-Rechte eingeräumt werden, was zu erheblichen Unterschieden zwischen den untersuchten Städten führt. Darüber hinaus stehen sie gegebenenfalls auch mit anderen Interessengruppen in Konflikt, was ihre Spielräume weiter einengen kann, aber in diesem Beitrag nicht im Fokus stand. Vor allem in den freiwilligen Aufgaben der Kommunen bestehen aufgrund der „Allzuständigkeit“ der Kommunen für lokale Sozialpolitik gute Voice-Möglichkeiten für zivilgesellschaftliche Interessenorganisationen wie Seniorenvertreter, was auch die oben angeführten Beispiele überwiegend zeigen. Bei Pflichtaufgaben hängt der Spielraum für kommunale Interessenvertretung dagegen von den variierenden Maßgaben des Gesetzgebers ab.

Seniorenbezogene kommunale Sozialpolitik beinhaltet oft eher kleinere Maßnahmen, die jedoch einen erheblichen Effekt auf die Lebensqualität der Betroffenen haben und im Stadtparlament und der Verwaltung durchaus umstritten sein können (Eifert 2006, S. 269). Die Ergebnisse des Beitrages zeigen, dass Seniorenvertretungen in praktisch allen Senioren betreffenden Bereichen kommunaler Sozialpolitik Voice ausüben. Ihren Schwerpunkt haben die beobachteten Fälle klar im Bereich der kommunalen Infrastrukturmaßnahmen, weniger häufig üben die Seniorenvertreter Voice in den Bereichen Qualität sozialer Dienstleistungen, Planungen, Preisen sozialer Dienstleistungen und Beratungsleistungen aus.

Voice ist aufwendig und in ihrem Erfolg unsicher (Hirschman 1970; Crouch 1995). Die Ergebnisse dieser Studie bestätigen das insofern, als dass das Verhinderungspotenzial kommunaler Seniorenvertretungen eher gering ist. Für eine stärkere Einflussnahme fehlen ihnen die formalen Rechte zur Mitentscheidung, was aus demokratietheoretischer Perspektive gute Gründe hat. Anders als in den Ländern und im Bund, erscheint darüber hinaus der öffentliche Diskurs aufgrund seiner geringeren Bedeutung in den Kommunen für die Einflussnahme weniger nutzbar. Wenn es ihnen aber gelingt, in Verwaltung und Politik hinreichend Unterstützung zu finden, können sie, wie die Fälle zu den Begegnungsstätten in den Kommunen O 1 und O 2 zeigen, ihre Interessen durchaus durchsetzen. Die Ergebnisse der Studie legen nahe, dass die Stärke von Seniorenvertretungen als Sprachrohr der
Nutzerinnen und Nutzer in ihrer Bürgerrolle darin liegt, neue Impulse in die kommunale Sozialpolitik einzubringen. Dies erscheint besonders erfolgreich, wenn der Gegenstand zu den freiwilligen Aufgaben kommunaler Sozialpolitik gehört. Für die Umsetzung sind sie aber stets auf die Unterstützung der kommunalen Verwaltung und Politik angewiesen.

\section{LITERATUR}

Alemann, U. v. (2000): Bedeutung vorparlamentarischer Beteiligungsformen für die kommunale Demokratie, in: BMFSFJ (Hrsg.): a.a.O., S. 37-50

Backhaus-Maul, H. (1994): Kommunale Sozialpolitik, in: Wollmann, H./Roth, R. (Hrsg.): Kommunalpolitik: Politisches Handeln in den Gemeinden, Bonn, S. 527-537

Backhaus-Maul, H. (1998): Kommunale Sozialpolitik, Sozialstaatliche Garantien und die Angelegenheiten der örtlichen Gemeinden, in: Wollmann, H./Roth, R. (Hrsg.): Kommunalpolitik. Politisches Handeln in den Gemeinden, Bonn, S. $689-702$

Bode, I. (2005): Einbettung und Kontingenz. Wohlfahrtsmärkte und ihre Effekte im Spiegel der neueren Wirtschaftssoziologie, in: Zeitschrift für Soziologie 34 (4), S. 250-269

Bundesministeriums für Familie, Senioren, Frauen und Jugend (BMFSFJ) (Hrsg.) (2000): Seniorenvertretungen. Verantwortung für das Gemeinwesen, Stuttgart

Burchardt, S. (2007): Veränderungspotenziale durch Governance in der kommunalen Jugendhilfe? Problemlagen und Steuerungsstrategien, in: Deutsches Jugend Institut, Projektgruppen E\&C und LOS (Hrsg.): Governancestrategien und lokale Sozialpolitik, S. 5-12

Clarke, J. (2006): Consumers, clients or citizens? Politics, Policy and practice in the reform of social care, in: European Societies 8 (3), S. 423-442 Clarke, J./Newman, J. (2007): What's in a name? New Labour's citizen-consumers and the remaking of public services, in: Cultural Studies 21 (4-5), S. $738-757$

Crouch, C. (1995): Exit or Voice: Two Paradigms for European Industrial Relations after the Keynesian Welfare State, in: European Journal of Industrial Relations 1 (1), S. 63-81

Dahme, H.-J/Wohlfahrt, N. (2011a): Kommunale Sozialpolitik - neue Herausforderungen, neue Konzepte, neue Verfahren, in: Dahme, H.-J./Wohlfahrt, N. (Hrsg.): Handbuch Kommunale Sozialpolitik, Wiesbaden, S. 9-18

Dahme, H.-J./Wohlfahrt, N. (2011b): Bürgerschaftliche Sozialpolitik, in: Dahme, H.-J./Wohlfahrt, N. (Hrsg.): Handbuch Kommunale Sozialpolitik, Wiesbaden, S. $395-408$

Eichler, M./Pfau-Effinger, B. (2009): The 'Consumer Principle' in the Care of Elderly People: Free Choice and Actual Choice in the German Welfare State, in: Social Policy \& Administration 43 (6), S. 617-633

Eifert, B. (2006): Seniorenvertretungen in Nordrhein-Westfalen als Beispiel politischer Partizipation älterer Menschen, in: Schröter, K. R./Zängl, P. (Hrsg.): Altern und bürgerschaftliches Engagement. Aspekte der Vergemeinschaftung und Vergesellschaftung in der Lebensphase Alter, Wiesbaden, S. 261-283 Evers, A./Heinze, R. G. (Hrsg.) (2008): Sozialpolitik. Ökonomisierung und Entgrenzung, Wiesbaden

Haarmann, A./KIenk, T./Weyrauch, P. (2010): Exit, Choice - and What About Voice? Public involvement in corporatist healthcare states, in: Public Management Review 12 (2), S. 213-231

Hirschman, A. O. (1970): Exit, voice, and loyalty. Responses to decline in firms, organizations, and states, Cambridge, Mass.

Klie, T./Pfundstein, T. (2008): Von der kommunalen Altenhilfeplanung zum Kultur- und Systemmanagement. Die neue Rolle der Kommunen in der Seniorenpolitik, in: Informationsdienst Altersfragen 35 (3), S. 7-10

Knemeyer, F.-L. (1995): Bürgerbeteiligung und Kommunalpolitik. Eine Einführung in die Mitwirkungsrechte von Bürgern auf kommunaler Ebene, München Künemund, H. (2001): Gesellschaftliche Partizipation und Engagement in der zweiten Lebenshälfte. Empirische Befunde zuTätigkeitsformen und Prognosen ihrer zukünftigen Entwicklung, Berlin

Le Grand, J. (1991): Quasi-markets and social policy, in: Economic Journal 101 (408), S. $1256-1267$

Mayer, K.-H. (2000): Seniorenvertretungen als politische Interessenvertretung der älteren Generation, in: BMFSFJ (Hrsg.): a.a.O., S. 16-23 
Mayring, P. (2000): Qualitative Inhaltsanalyse, in: Forum Qualitative Sozialforschung 1 (2) Art. 20, http://nbn-resolving.de/urn:nbn:de:0114-fqs0002204

Mayring, P. (2008): Qualitative Inhaltsanalyse. Grundlagen und Techniken,

Weinheim

Naegele, G. (1999): The politics of old age in Germany, in: Walker, A./Naegele,

G. (Hrsg.): The politics of old age in Europe, Buckingham/Philadelphia,

S. 93-109

Pabst, S. (2002): Implementation sozialpolitischer Programme im Föderalismus. Pflegepolitischer Wandel in Ländern und Kommunen mit Einführung der Pflegeversicherung, Berlin

Pitschas, R. (2000): Rechtliche Absicherung und Einflussmöglichkeiten von Senioren, in: BMFSFJ (Hrsg.): a.a.O., S. 130-134

Pfau-Effinger, B./Eichler, M./Och, R. (2012): Erklärung der Differenzen in der lo kalen Sozialpolitik zur Pflege älterer Menschen in West- und Ostdeutschland, in: Soeffner, G. (Hrsg.): Beiträge zum 35. Soziologentag in Frankfurt/M., Frankfurt a. M. (im Erscheinen)

Rokkan, S. (1974): Entries, voices, exits:Towards a possible generalization of the Hirschman model, in: Social Science Information 13 (1), S. 39-53

Roth, R. (1997): Die Kommune als Ort der Bürgerbeteiligung, in: Klein, A./ Schmalz-Bruhns, R. (Hrsg.): Politische Beteiligung und Bürgerengagement in Deutschland. Möglichkeiten und Grenzen, Baden-Baden, S. 404-447

Roth, R. (1999): Kommunale Sozialstaatlichkeit als Perspektive für eine notwendige Neuorientierung sozialer Arbeit, in: Rundbrief Gilde Soziale Arbeit (2), S. 3-14
Schmidt, M. G. (2009): Altern und politische Partizipation, in: Kocka, J./Staudinger, U. M. (Hrsg.): Altern: Familie, Zivilgesellschaft, Politik, Stuttgart, S. $273-286$

Vabø, M. (2006): Caring for people or caring for proxy consumers?, in: European Societies 8 (3), S. 403-422

Vanselow, H. (2000): Mitwirkung und Mitbestimmung bei politischen Entscheidungen von Seniorenvertretungen, in: BMFSFJ (Hrsg.): a.a.O., S. 121-125

\section{AUTOR}

RALF OCH, Diplom Sozialwissenschaftler, ist wissenschaftlicher Mitarbeiter am Fachbereich für Sozialwissenschaften an der Universität Hamburg. Arbeitsschwerpunkte: lokale Sozialpolitik, vergleichende Wohlfahrtstaatsforschung, Zivilgesellschaft.

@ralf.och@wiso.uni-hamburg.de 